\title{
Dinâmica de serapilheira em uma área de floresta de terra firme, Amazônia Ocidental
}

\author{
Gabriel Araújo Paes FREIRE ${ }^{1 *}$, Dione Judite VENTURA², Igor Georgios FOTOPOULOS ${ }^{3}$, \\ Diogo Martins ROSA ${ }^{4}$, Renata Gonçalves AGUIAR ${ }^{5}$, Alessandro Carioca de ARAÚJO ${ }^{6}$
}

\author{
${ }^{1}$ Programa de Pós-Graduação em Ciências Ambientais, Universidade Federal do Rondônia, Rolim de Moura, RO, Brasil. \\ 2Instituto Nacional de Pesquisas Espaciais, São José dos Campos, SP, Brasil. (ORCID: 0000-0003-3457-8399) \\ ${ }^{3}$ Departamento de Biologia, Universidade Federal de Rondônia, Porto Velho, RO, Brasil. (ORCID: 0000-0002-7726-0703) \\ ${ }^{4}$ Secretaria de Estado do Desenvolvimento Ambiental, Porto Velho, RO, Brasil. (ORCID: 0000-0003-3427-8353) \\ ${ }_{5}^{5}$ Departamento de Engenharia Ambiental, Universidade Federal de Rondônia, Ji-Paraná, RO, Brasil. (ORCID: 0000-0003-1431-912X) \\ ${ }^{6}$ Embrapa Amazônia Oriental, Belém, PA, Brasil. (ORCID: 0000-0002-7361-5087) \\ *E-mail: paesfreire@gmail.com (ORCID: 0000-0003-4678-9126)
}

Recebido em 01/10/2019; Aceito em 11/04/2020; Publicado em 24/04/2020.

\begin{abstract}
RESUMO: O objetivo deste estudo foi avaliar a produção e decomposição de serapilheira em uma área de floresta primária de terra firme na Reserva Biológica do Jaru. Para tanto, foi implantada uma parcela permanente de 1 hectare, onde foram demarcadas 25 subparcelas com 20 × $20 \mathrm{~m}$ cada. Para a coleta de serapilheira, instalou-se 25 coletores de PVC (1 em cada centro da subparcela), medindo $0,25 \mathrm{~m}^{2}$, a 1 metro do solo, com malha de nylon de $1 \mathrm{~mm}$. Para estimar o estoque de serapilheira, utilizou-se 25 coletores de madeira, medindo $0,25 \mathrm{~m}^{2}$, com malha de nylon de $1 \mathrm{~mm}$, dispostos no solo de cada subparcela. As coletas da pesquisa foram realizadas quinzenalmente, entre os meses de outubro de 2016 a setembro de 2017, e a serapilheira triada nas frações: folha, galho, material reprodutivo e miscelânea. No laboratório, os materiais amostrados foram secos em estufa à $80^{\circ} \mathrm{C}$ e pesados em balança de precisão centesimal, sendo que a decomposição de serapilheira foi estimada através da relação entre produção e estoque. De acordo com os resultados obtidos, a produção de serapilheira total foi de $14,13 \mathrm{Mg} \mathrm{ha}^{-1}$ e as frações seguiram o padrão: folha $>$ miscelânea $>$ galho $>$ material reprodutivo. As maiores produções aconteceram na estação seca e a taxa de decomposição foi 1,37, indicando que a atividade microbiana na área de estudo é acelerada.
\end{abstract}

Palavras-chave: dinâmica sazonal; nutrição de plantas; ecologia florestal; decomposição.

\section{Litter dynamics in a forest area of ground firm, Western Amazon}

\begin{abstract}
The objective of this study was to evaluate the litter production and decomposition in an area of primary upland forest in the Jaru Biological Reserve. For that, a permanent plot of 1 hectare was implanted, where 25 subplots with $20 \times 20$ m each were demarcated. For the collection of litter, 25 PVC collectors were installed (1 in each center of the subplot), measuring $0.25 \mathrm{~m}^{2}, 1$ meter from the ground, with $1 \mathrm{~mm}$ nylon mesh. To estimate the litter stock, 25 wood collectors, measuring $0.25 \mathrm{~m}^{2}$, with $1 \mathrm{~mm}$ nylon mesh, were used, arranged in the soil of each subplot. The collections of the research were carried out fortnightly, between the months of October 2016 and September 2017, and the litter is sorted in the fractions: leaf, branch, reproductive material and miscellaneous. In the laboratory, the sampled materials were dried in an oven at $80{ }^{\circ} \mathrm{C}$ and weighed on a centesimal precision scale, and the litter decomposition was estimated through the relationship between production and stock. According to the results obtained, the total litter production was $14.13 \mathrm{Mg} \mathrm{ha}{ }^{-1}$ and the fractions followed the pattern: leaf $>$ miscellaneous $>$ branch $>$ reproductive material. The highest yields occurred in the dry season and the decomposition rate was 1.37 , indicating that the microbial activity in the study area is accelerated.

Keyword: seasonal dynamics; plant nutrition; forest ecology; decomposition.
\end{abstract}

\section{INTRODUÇÃO}

A manutenção do ecossistema está associada com a ciclagem de nutrientes estocados na vegetação, solo e serapilheira (BARBOSA et al., 2017). Denomina-se serapilheira a matéria orgânica depositada no solo da floresta, como folhas, ramos, cascas, sementes, frutos, flores, gravetos e fragmentos vegetais não identificados, em diferentes etapas de decomposição (SILVA et al., 2014). A serapilheira é o mais importante estágio de transferência de energia e de fluxo de nutrientes para o solo. Ela se movimenta e se transforma nos ecossistemas florestais através do ciclo biogeoquímico, o qual é necessário para processar as reações metabólicas nas diferentes formas de vida. A reciclagem dos nutrientes da serapilheira no solo é feita pelos decompositores e pela rápida absorção das raízes das plantas, mediante a associação simbiótica com fungos para incremento da biomassa vegetal. (HOLANDA et al., 2017; MARTINS, 2012) Não obstante, a ciclagem de nutrientes favorece o desenvolvimento e a manutenção da comunidade florística (SANCHES et al., 2009), além de contribuir com o fluxo de carbono no ambiente (AN et al., 2017).

Todavia, a produção de serapilheira pode ser influenciada por diversos fatores, especialmente pelas condições edafoclimáticas, como: altitude, latitude, precipitação, 
temperatura, luminosidade, vento, estágio sucessional, disponibilidade hídrica, entre outras características do ecossistema (HOLANDA et al., 2017). Tais aspectos podem levar a sazonalidade da deposição do material orgânico no terreno.

Nesse sentido, o acúmulo da biomassa aérea no solo contribui para a formação de um horizonte orgânico que protege as características edáficas. Isto mantém as estruturas do solo e possibilita maior retenção de umidade, aeração e redução do processo erosivo, atuando como um isolante térmico. Este acúmulo no piso florestal está diretamente ligado com a atividade decompositora dos microrganismos, que supre o solo com nutrientes e restauram a fertilidade do ecossistema (SANCHES et al., 2009).

Além disso, as florestas tropicais apresentam vasta biodiversidade que fornece à serapilheira uma composição rica e constante de nutrientes, favorecendo a manutenção estrutural das comunidades, bem como o desenvolvimento dinâmico dos ecossistemas (SANCHES et al., 2009).

Assim, estudos que buscam compreender o funcionamento de ambientes florestais, em distintas condições edafoclimáticas, são de grande relevância para elucidar os fatores associados com a produção de serapilheira nas províncias fitogeográficas da Amazônia.

No rastreio disso, tem-se que os ecossistemas amazônicos apresentam solos com baixa fertilidade e para que a flora se desenvolva, é necessária a intensa ciclagem de nutrientes através da decomposição da matéria orgânica. Por este motivo, os coeficientes de decomposição (K) encontrados na região variam em torno 1,35 (SANCHES et al, 2009) e 1,6 (MARTINS et al., 2018b).

Contudo, a grande extensão da Amazônia tem dificultado caracterizar os padrões de produção e decomposição de serapilheira (ALMEIDA et al., 2015), e diante disto destacase a necessidade de espacializar o conhecimento acerca da ciclagem de nutrientes para a conservação e o manejo eficiente das florestas regionais.

Por esta razão, buscou-se analisar nesse estudo como a dinâmica de produção de serapilheira (total e fracionada) está correlacionada com as variáveis climáticas de temperatura e umidade relativa do ar, bem como estimar qual a velocidade da decomposição de serapilheira em um trecho de floresta primária de terra firme na Reserva Biológica do Jaru/RO.

\section{MATERIAL E MÉTODOS}

2.1. Área de estudo

O estudo foi conduzido na Reserva Biológica do Jaru (Rebio Jaru), 10¹1'11,4”S; 6152'29,9”W, área localizada no Estado de Rondônia (Figura 1).

A Rebio Jaru é uma unidade de conservação federal de proteção integral, sob tutela do Instituto Chico Mendes de Conservação da Biodiversidade (ICMBio). De acordo com a fasciação da vegetação e pluviosidade da região, a altura média do dossel da floresta é de 33 metros, e a precipitação anual da área de 1.989,65 mm (ANDRADE et al., 2009).

Para a presente pesquisa foi instalada uma parcela permanente de $100 \times 100 \mathrm{~m}$ (1 ha), dividida em 25 subparcelas com $20 \times 20 \mathrm{~m}$ cada, localizada no sentido noroeste e a montante da torre de observação meteorológica do Experimento de Grande Escala da Biosfera-Atmosfera na Amazônia - Programa LBA.

\subsection{Serapilheira produzida e acumulada}

No centro de cada subparcela foi implantado um coletor de serapilheira (Figura 2), medindo 0,50 x 0,50 m (0,25 $\left.\mathrm{m}^{2}\right)$, feito em PVC, com um fundo de tela de nylon de $1 \mathrm{~mm}$ de malha a $1 \mathrm{~m}$ do solo.

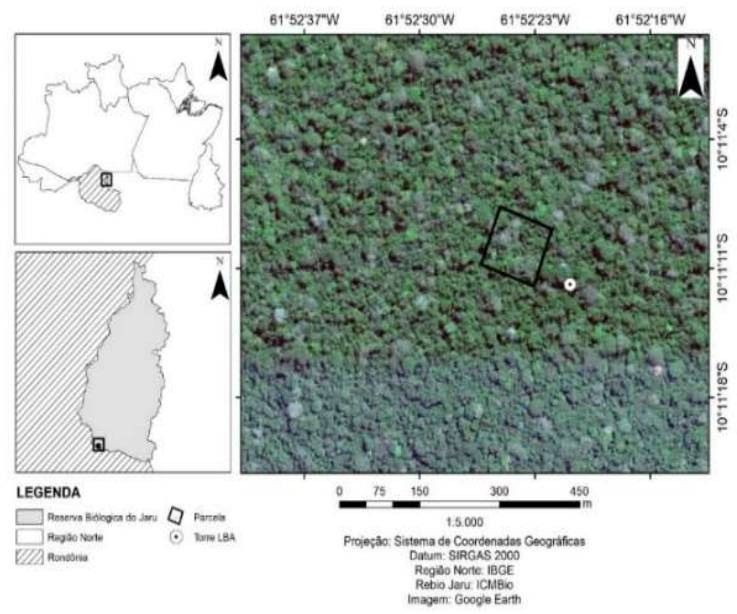

Figura 1. Localização da área de estudo na Reserva Biológica do Jaru, Amazônia Ocidental, Rondônia.

Figure 1. Location of the study area in the Jaru Biological Reserve, Rondônia, Western Amazon.

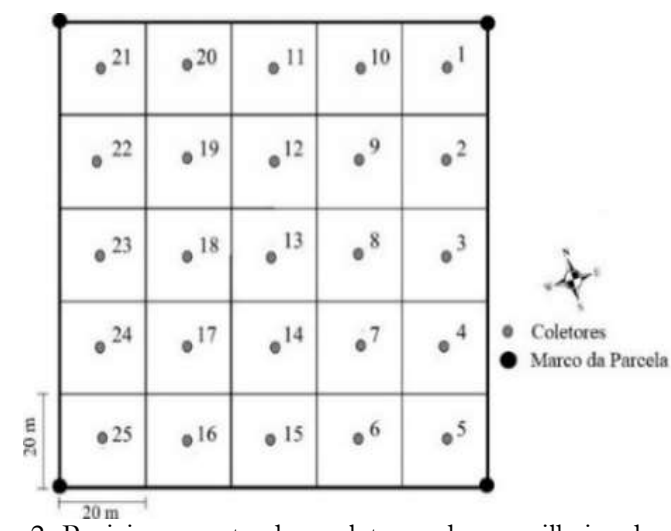

Figura 2. Posicionamento dos coletores de serapilheira dentro das subparcelas na Reserva Biológica do Jaru (Rebio Jaru), Amazônia Ocidental.

Figure 2. Positioning of litter collectors within the subplots in the Jaru Biological Reserve (Rebio Jaru), Western Amazon.

As coletas de serapilheira foram realizadas quinzenalmente, no período de outubro de 2016 a setembro de 2017. O material de amostragem foi acondicionado em sacos plásticos devidamente identificados em campo, e, posteriormente, levado para laboratório onde secaram em estufa à $80{ }^{\circ} \mathrm{C}$ com ventilação forçada até obter massa constante. Após a secagem foi realizada a triagem da serapilheira em frações de folha, galho, material reprodutivo (flor, fruto e semente) e miscelânea (material não identificado, casca, fezes e fauna). Em seguida, foi realizada a pesagem em balança de precisão centesimal e as massas de serapilheira convertidas para $\mathrm{Mg} \mathrm{ha}{ }^{-1}$, sendo estas quantificadas mensalmente.

As amostras do estoque de serapilheira foram coletadas utilizando 25 molduras quadradas de madeira, contendo 0,5 $\mathrm{m}$ de lado e $0,25 \mathrm{~m}^{2}$, distribuídas, sistematicamente, próximas dos coletores de serapilheira (Figura 3). A coleta foi realizada trimestralmente, no período de outubro de 2016 a setembro 
de 2017. Foi considerado como serapilheira acumulada todo o material decíduo depositado sobre a moldura no solo e em todos os graus de decomposição (galhos com diâmetros superiores a $2 \mathrm{~cm}$ de diâmetro foram descartados). O material coletado foi acondicionado em sacos plásticos e identificados em campo, adotando o mesmo procedimento de secagem e pesagem utilizado na amostragem de serapilheira.

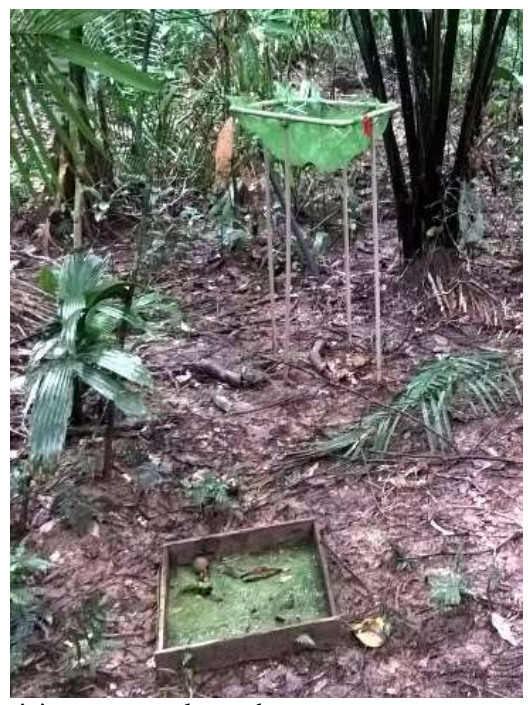

Figura 3. Posicionamento dos coletores.

Figure 3. Positioning of the collectors.

\subsection{Caracterização química da serapilheira}

Os teores de macronutrientes (nitrogênio, fósforo, potássio, cálcio, magnésio e enxofre) foram analisados seguindo a metodologia proposta pela Embrapa (2009).

\subsection{Estimativa do coeficiente de decomposição}

O coeficiente de decomposição $(\mathrm{K})$, proposto por Olson (1963), foi estimado aplicando a Equação 1.

$$
\mathrm{K}=\mathrm{L} \mathrm{X}^{-1}
$$

em que: $\mathrm{K}=$ constante de decomposição; $\mathrm{L}=$ serapilheira produzida durante $\mathrm{o}$ ano $\left(\mathrm{Mg} \mathrm{ha}^{-1}\right) ; \mathrm{X}^{-1}=$ média anual do estoque de serapilheira acumulada sobre o solo $\left(\mathrm{Mg} \mathrm{ha}^{-1}\right)$.

A partir do valor do $K$, também foi calculado o tempo médio de renovação de serapilheira no solo (em anos) ( $\left.\mathrm{K}^{\prime}\right)$, utilizando a Equação 2, proposta por Hopkins (1966).

$$
\mathrm{K}^{\prime}=1 \mathrm{~K}^{-1}
$$

em que: $\mathrm{K}^{-1}=$ Inverso da constante da decomposição

Para realizar os cálculos do tempo necessário para a decomposição de $50 \%(\mathrm{t}=0,5)$ e $95 \%\left(\mathrm{t}==_{0,05}\right)$ da serapilheira, utilizaram-se a Equação 3 e 4, respectivamente, proposta por Shanks; Olson (1961):

$$
\begin{aligned}
\mathrm{t}_{0,5} & =-\ln 0,5 \mathrm{~K}^{-1} \\
\mathrm{t}_{0,05} & =3 \mathrm{~K}^{-1}
\end{aligned}
$$

\subsection{Coleta de dados meteorológicos}

Os dados de temperatura do ar (Termohigrômetro HC2S3 - Vaisala, Helsinki, FIN), precipitação (pluviômetro
TB4-L) e umidade relativa do ar (Termohigrômetro HC2S3 - Vaisala, Helsinki, FIN) utilizados nesse estudo, foram obtidos da torre micrometeorológica do Programa LBA presente na Rebio Jaru.

Através de sensores acoplados na torre, os dados foram coletados em intervalos de 30 segundos e suas médias geradas a cada 30 minutos, automaticamente armazenadas em um datalogger CR1000 (Campbell Scientific Instrument, Utah, USA). Posteriormente, foi realizada a certificação e o aproveitamento dos dados para excluir possíveis falhas, e calcular a média mensal para os dados de temperatura do ar, umidade relativa do ar e precipitação.

\subsection{Análise dos dados}

Para a determinação das correlações entre a produção total de serapilheira e dos seus componentes (folha, miscelânea, galho, e material reprodutivo) com as variáveis meteorológicas (precipitação, umidade relativa do ar e temperatura do ar), utilizou-se o coeficiente de correlação de Pearson $(\alpha=0,05)$

\section{RESULTADOS}

\subsection{Serapilheira total}

No período da pesquisa, a produção total de serapilheira foi de $14,13 \mathrm{Mg} \mathrm{ha}^{-1} \mathrm{ano}^{-1}$. A maior produção de biomassa acorreu no mês de setembro $\left(2,45 \mathrm{Mg} \mathrm{ha}^{-1}\right)$, caracterizado como o fim do período seco, seguido do mês de agosto, com $2,27 \mathrm{Mg} \mathrm{ha}^{-1}$. As menores deposições foram registradas nos meses de fevereiro e março, com 0,47 e 0,35 $\mathrm{Mg} \mathrm{ha}{ }^{-1}$, respectivamente.

Através do coeficiente de correlação de Pearson (Tabela 1), observa-se uma forte correlação negativa entre a produção total de serapilheira e a precipitação $(\mathrm{r}=-0,67, \varrho$-valor $=$ 0,05).

Com relação a temperatura, a serapilheira total manteve uma correlação positiva $(\mathrm{r}=0,67, \varrho$-valor $=0,02)$, e negativa com a umidade relativa do ar $(\mathrm{r}=-0,89, \mathrm{Q}$-valor $<0,001)$.

\subsection{Serapilheira fracionada}

De acordo com a amostragem total de serapilheira, a fração foliar apresentou maior produção, com 8,64 $\mathrm{Mg} \mathrm{ha}^{-}$ 1 ano $^{-1}(61,15 \%)$. Seguido da fração miscelânea, cuja produção foi $2,65 \mathrm{Mg} \mathrm{ha}^{-1}$ ano $^{-1}(18,73 \%)$. Em terceiro registrou-se a produção da fração galho, com $1,70 \mathrm{Mg} \mathrm{ha}^{-1}$ $\mathrm{ano}^{-1}(12,03 \%)$ e maior incremento no período chuvoso amazônico. Em último foi fixada a produção de material reprodutivo (flor, fruto e semente), com $1,15 \mathrm{Mg} \mathrm{ha}^{-1} \mathrm{ano}^{-1}$ $(8,11 \%)$.

\subsection{Nutrientes produzidos pela serapilheira total}

$\mathrm{Na}$ Tabela 2, é vista a concentração de nutrientes produzidos pela serapilheira total nos períodos chuvoso e seco. De modo geral, os nutrientes obedeceram a seguinte ordem: $\mathrm{N}>\mathrm{Ca}>\mathrm{K}>\mathrm{Mg}>\mathrm{P}>\mathrm{S}$, tanto no período chuvoso quanto no período seco.

\subsection{Taxa de decomposição de serapilheira}

No presente estudo, o estoque de serapilheira alcançou uma produção de $10,31 \mathrm{Mg} \mathrm{ha}^{-1} \mathrm{ano}^{-1}$. Sendo que o coeficiente de decomposição da serapilheira $(K)$ foi de 1,37 e tempo médio para a renovação do estoque ( $\left.K^{\prime}\right)$ no solo de 0,73 ano (266 dias). Não obstante, o tempo médio estimado 
para a decomposição de $50 \%$ de serapilheira foi de 0,51 ano, ou seja, 184 dias. E o tempo médio estimado para a decomposição de $95 \%$ de serapilheira foi de 2,19 anos, ou 799 dias.

Tabela 1. Teste de correlação entre as frações de serapilheira da Reserva Biológica do Jaru, Rondônia, e suas variáveis micrometeorológicas. Table 1. Correlation test between the litter fractions of Jaru Biological Reserve, Rondônia, and its micrometeorological variables.

\begin{tabular}{|c|c|c|c|c|c|c|}
\hline \multirow{2}{*}{ Fração } & \multicolumn{2}{|c|}{ Temperatura } & \multicolumn{2}{|c|}{ Umidade Relativa } & \multicolumn{2}{|c|}{ Precipitação } \\
\hline & $r$ & $p$-valor & $r$ & $p$-valor & $r$ & p-valor \\
\hline Folha & $0,57^{*}$ & $0,05^{*}$ & $-0,97 *$ & $<0,001 *$ & $-0,80$ & 0,08 \\
\hline Galho & $0,59 *$ & $0,04 *$ & $-0,45$ & 0,14 & $0,00 *$ & $0,05^{*}$ \\
\hline MR & 0,55 & 0,06 & $-0,36$ & 0,25 & $0,07^{*}$ & $<0,001 *$ \\
\hline Misc. & $0,59 *$ & $0,04^{*}$ & $-0,44$ & 0,15 & $-0,12$ & 0,15 \\
\hline Total & $0,67 *$ & $0,02^{*}$ & $-0,89 *$ & $<0,001^{*}$ & $-0,67^{*}$ & $0,05^{*}$ \\
\hline
\end{tabular}

MR: Material Reprodutivo; Misc.: Miscelânea; *Significativos Estatisticamente

Tabela 2. Teores e conteúdos de nutrientes da serapilheira produzida nas estações chuvosa e seca na Reserva Biológica do Jaru, Rondônia. Table 2. Nutrient stock and contents of the litter produced in the rainy and dry seasons in Jaru Biological Reserve, Rondônia

\begin{tabular}{|c|c|c|c|c|c|c|}
\hline \multirow{2}{*}{ Nutrientes } & \multicolumn{2}{|c|}{ Teor $\left(\mathrm{g} \mathrm{kg}^{-1}\right)$} & \multirow{2}{*}{$\begin{array}{l}\text { Proporção do Teor }(\%) \\
\text { Chuvoso/Seco }\end{array}$} & \multicolumn{2}{|c|}{ Conteúdo $\left(\mathrm{kg} \mathrm{ha}^{-1}\right)$} & \multirow{2}{*}{$\begin{array}{c}\text { Proporção do Conteúdo }(\%) \\
\text { Chuvoso/Seco }\end{array}$} \\
\hline & Chuvoso & Seco & & Chuvoso & Seco & \\
\hline Nitrogênio $(\mathrm{N})$ & 19,1 & 19,6 & 97,4 & 109,9 & 164,1 & 67,0 \\
\hline Cálcio (Ca) & 5,2 & 7,7 & 67,5 & 29,9 & 64,4 & 46,4 \\
\hline Potássio (K) & 4,2 & 6,4 & 65,6 & 24,1 & 53,6 & 45,1 \\
\hline Magnésio (Mg) & 1,4 & 2,3 & 60,9 & 8,0 & 19,2 & 41,9 \\
\hline Fósforo $(\mathrm{P})$ & 1,0 & 0,8 & 125,0 & 5,7 & 6,7 & 86,0 \\
\hline Enxofre (S) & 1,0 & 1,6 & 62,5 & 5,7 & 13,4 & 43,0 \\
\hline
\end{tabular}

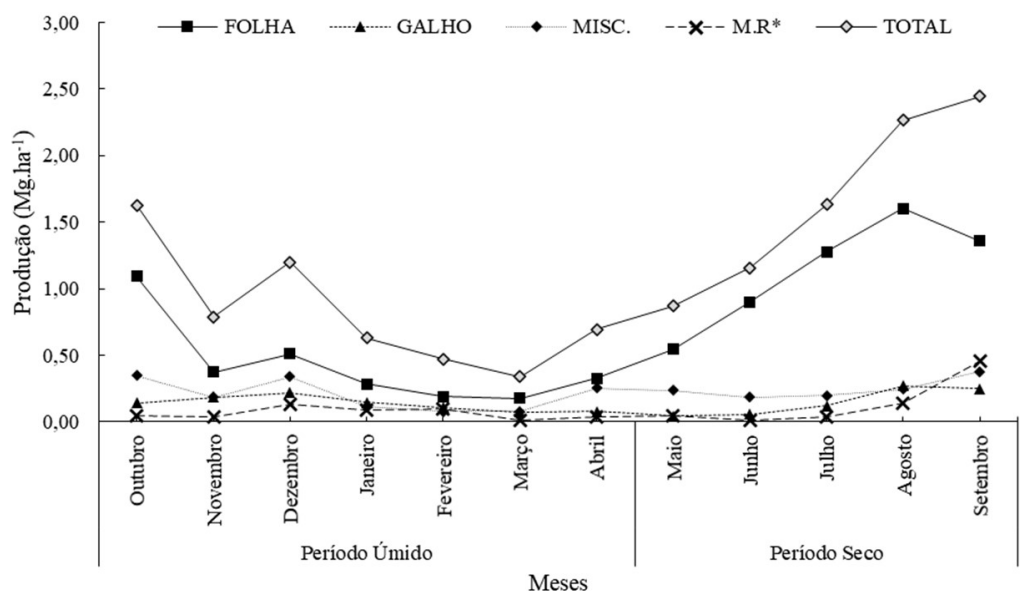

Figura 3. Média mensal da produção de serapilheira fracionada $\left(\mathrm{Mg} \mathrm{ha}^{-1}\right)$ no período de outubro de 2016 a setembro de 2017 na Reserva Biológica do Jaru, Rondônia. Misc.: Miscelânea; M.R*: Material Reprodutivo.

Figure 3. Monthly average of fractionated litter production $\left(\mathrm{Mg} \mathrm{ha}^{-1}\right)$ from October 2016 to September 2017 in Jaru Biological Reserve, Rondônia.

\section{DISCUSSÃO}

A quantidade de serapilheira registrada na presente pesquisa está dentro dos padrões descritos para a Amazônia Ocidental, sugerindo que a produção média de serapilheira nesta província fitogeográfica é superior a $10,5 \mathrm{Mg} \mathrm{ha}^{-1} \mathrm{ano}^{-1}$ (ANDRADE et al., 1999). Valores acima e abaixo ao exposto nesse estudo, foram observados em outras localidades da Amazônia, a exemplo da taxa de serapilheira quantificada por Barlow et al. (2007) numa vegetação primária $\left(12,4 \mathrm{Mg} \mathrm{ha}^{-1}\right.$ ano $\left.{ }^{-1}\right)$ e secundária $\left(13,4 \mathrm{Mg} \mathrm{ha}^{-1}\right.$ ano $\left.^{-1}\right)$ no estado do Pará, e por Ourique et al. (2016) em uma área de floresta no Amazonas (6,3 $\left.\mathrm{Mg} \mathrm{ha}^{-1} \mathrm{ano}^{-1}\right)$.

Embora esse estudo apresente uma produção acima da média quando comparado com outros trabalhos desenvolvidos na Amazônia, é válido destacar que o fenômeno climático do El Niño ocorreu de forma acentuada na região em 2015 e meados de 2016 (LEITOLD et al., 2018). Tal fenômeno provoca eventos de seca extrema na Amazônia e, como resposta, as plantas tendem a perder estruturas vegetais para a economia de água nos tecidos vivos (COSTA et al., 2014). Porém, a vegetação pode demorar a dar respostas fisiológicas em termos de máxima produção de serapilheira, fator conhecido como delay de produção, comumente observado nos períodos de transição sazonal da Amazônia (BARBOSA et al., 2017).

Todavia, foi constatado que a produção de serapilheira manteve um padrão sazonal, com maior produção no período seco e menor produção no período chuvoso. $\mathrm{O}$ mesmo padrão de produção foliar, com máxima deposição no período seco e mínima produção no período chuvoso foi observado em outras regiões da Amazônia (MOURA et al., 2016; PEREIRA et al., 2017). Com base nisso, é possível deduzir que os níveis de serapilheira no ambiente amazônico são influenciados pelo balanço hídrico.

A maior produção de folhas na região amazônica acontece pela renovação no período seco devido ao déficit 
hídrico (MARTINS et al., 2018a), associado também à renovação foliar para aproveitamento da radiação fotossintéticamente ativa (KIM et al., 2012). Isto é possível de explicar pela senescência característica das espécies arbóreas amazônicas, em resposta a processos metabólicos e ou estímulos do ambiente, como umidade relativa do ar $(r=0,80$ e $\varrho$-valor $=0,05)$ e precipitação $(\mathrm{r}=-0,87$ e $\varrho-$ valor $=<0,001)$.

Com relação a produção da fração miscelânea, as diferentes taxas observadas em estudos desenvolvidos num mesmo bioma podem não estar atrelados somente a fatores bióticos e abióticos, mas também a questões associadas com o tempo de estudo ou ruptura e decomposição das diferentes frações de serapilheira. Assim, durante a quantificação do material identificado pelo pesquisador, distintas circunstâncias podem influenciar na determinação dos valores.

No entanto, a elevada produção de galhos na época chuvosa, provavelmente ocorreu devido a maior presença de ventanias e superior quantidade de água absorvida pelos vegetais nesse período, fazendo com que as galhadas tenham maior peso e atrito, resultando em sua quebra (SILVA et al., 2016). Não obstante, a queda de galhos no período seco pode ocorrer devido a ventos fortes e também pelo estresse hídrico, visto que as altas temperaturas podem fazer com que os galhos sequem e se rompam mais facilmente nessa estação.

$\mathrm{Na}$ análise da produtividade da fração de material reprodutivo, destaca-se que esta pode estar atrelada à adaptação da planta à sazonalidade climática, cujo incremento é frequentemente maior no fim da estação seca e início da estação úmida, considerados como os períodos mais propícios à germinação vegetal (ALMEIDA et al., 2015).

Nesse estudo, a produção total de serapilheira registrou a maior quantidade de nutriente para $\mathrm{N}$ e isto se deve a eficiente fixação deste elemento pelas plantas, demonstrando conformidade com os resultados vistos em outros trabalhos (GODINHO et al., 2013; CALDEIRA et al., 2013; ). A segunda maior taxa nutricional foi de $\mathrm{Ca}$, com picos mais elevados na estação seca, devido, supostamente, à menor taxa metabólica neste período fazendo com que reduza sua mobilidade (DIAS et al., 2002) e a sua liberação na serapilheira está diretamente ligada à decomposição biológica e posterior absorção direta das raízes finas (PEREIRA et al., 2016). Na sequência, tem-se $K$ que também foi mais expressivo no período seco, pelo fato deste elemento se encontrar nos vegetais apenas na sua forma iônica (GODINHO et al., 2013).

Não obstante, estas concentrações de nutrientes podem variar de acordo com as condições edafoclimáticas e pela presença de determinadas espécies florestais ou idades das plantas (HOLANDA et al., 2017). Além disto, a velocidade de decomposição da matéria orgânica e de disponibilidade dos nutrientes são influenciadas pela presença de oxigênio no solo, umidade, temperatura, assim como pela efetividade dos organismos decompositores, como bactérias, fungos, macro artrópodes e outros animais capazes de transformar a serapilheira em nutrientes no ecossistema (CASTRO et al., 2016).

Com base nisso, a manutenção dos sistemas florestais é realizada pela transferência de elementos da vegetação para o solo e por meio da produtividade primária líquida (ÇAKIR; AKBURAK, 2017). Por esta razão, a interação de fatores bióticos e abióticos são de fundamental importância, pois são estes que irão regular a decomposição de serapilheira no ambiente, fazendo com que sejam liberados os nutrientes para a manutenção das comunidades florísticas, onde valores de taxas de decomposição maiores que 1,1 representam alta decomposição e rápido aproveitamento dos nutrientes pela vegetação (GOLLEY, 1978). Contudo, a dinâmica da decomposição de serapilheira pode se tornar acelerada ou lenta, conforme determinados fatores ambientais, sendo que no presente estudo o principal contribuinte diz respeito a umidade do ecossistema, a qual, provavelmente, favoreceu a multiplicação dos microrganismos presentes no solo e consequentemente a decomposição da matéria orgânica. Por estes motivos, infere-se que no trecho de floresta investigada há uma rápida velocidade de decomposição e, também, rápida absorção de nutrientes pelos vegetais.

\section{CONCLUSÕES}

A serapilheira total presente no trecho de floresta investigada na Rebio Jaru obteve maior produção no período seco da Amazônia, sendo a fração de folhas a mais abundante independente dos meses analisados, o qual é típico das florestas tropicais. A serapilheira fracionada teve a seguinte ordem decrescente: folha $>$ material reprodutivo $>$ miscelânea $>$ galho. E o nitrogênio, maior aporte dentre os nutrientes avaliados.

Por fim, destaca-se a alta taxa de decomposição $(1,37)$ do estoque de serapilheira, o que denota uma rápida liberação dos nutrientes da matéria orgânica senescente que compõem o piso da floresta estudada.

\section{REFERÊNCIAS}

ALMEIDA, E. J., LUIZAO, F., RODRIGUES, D.J. Produção de serrapilheira em florestas intactas e exploradas seletivamente no sul da Amazônia em função da área basal da vegetação e da densidade de plantas. Acta Amazonica, Manaus, v. 45, n. 2, p. 157-166, 2015. DOI: http:/ /dx.doi.org/10.1590/1809-4392201402543

AN, J. Y.; PARK, B. B.; CHUN, J. H.; OSAWA, A. Litterfall production and fine root dynamics in cool-temperate forests. PLoS ONE, v. 12, n. 6, p. 1-16, 2017, DOI: https://dx.doi.org/10.1371/journal.pone.0180126

ANDRADE, A. G. de; CABALLERO, S. S. U.; FARIA, S. M. de. Ciclagem de nutrientes em ecossistemas florestais. Rio de Janeiro: Embrapa Solos, 1999. 50 p.

ANDRADE, N. L. R.; AGUIAR, R.G.; SANCHES, L.; ALVES, E. C. R. F.; NOGUEIRA, J. S. Participação no saldo de radiação em áreas de floresta amazônica e floresta de transição Amazônia-Cerrado. Revista Brasileira de Meteorologia, v. 4, n. 3, p. 346-355, 2009. DOI: http://dx.doi.org/10.1590/S010277862009000300008

BARBOSA, V.; BARRETO-GARCIA, P.; GAMARODRIGUES, E.; PAULA, A. Biomassa, Carbono e Nitrogênio na Serapilheira Acumulada de Florestas Plantadas e Nativa. Floresta e Ambiente, v. 24, p. 1-9, 2017. http://dx.doi.org/10.1590/2179-8087.024315

BARLOW, J.; GARDNER, T. A.; FERREIRA, L. V.; PERES, C. A. Litter fall, and decompositon in primary, secondary and plantation forests in the Brazilian Amazon. Forest Ecology and Management, Amsterdam, v. 247, n. 1-3, p. 91-97, 2007. DOI: https://dx.doi.org/10.1016/j.foreco.2007.04.017 
ÇAKIR, M.; AKBURAK, S. Litterfall and nutrientes return to soil in pure and mixed stands of oak and beech. Journal of the Faculty of Forestry Istanbul University, v. 67 , n. 2, p. 178-203. DOI: https://dx.doi.org/10.17099/jffiu.301602

CALDEIRA, M. V. W.; SILVA, R. D.; KUNZ, S. H.; ZORZANELLI, J. P. F.; CASTRO, K. C.; GODINHO, T. O. Biomassa e nutrientes da serapilheira em diferentes coberturas florestais. Comunicata Scientiae, Bom Jesus, v. 4, n. 2, p. 111-119, 2013.

CASTRO, R. M. S.; RUIVO, M. L. P.; SANTOS, S. F.; RODRIGUES, P. G. Influência do estresse hídrico sobre a decomposição da serapilheira em floresta amazônica de terra firme. Boletim do Museu Paraense Emílio Goeldi, Belém, v. 11, n. 3, p. 343-350, 2016.

COSTA, M. C.; COSTA, A. C. L.; COElHO, L. T. S.; SILVA, T. M. L.; AZEVEDO, A. F. Correlação entre precipitação pluviométrica e umidade do solo na produção de serapilheira em Caxiuanã (PA). Revista Ibero-Americana de Ciências Ambientais, v. 5, n. 1, p. 170-179, 2014. https:/ /dx.doi.org/10.6008/SPC21796858.2014.001.0012

DIAS, H. C. T.; FIGUEIRA, M. D.; SILVEIRA, V.; FONTES, M. A. L.; OLIVEIRA-FILHO, A. T.; SCOLFORO, J. R. F. Variação temporal de nutrientes na serapilheira de um fragmento de floresta estacional semidecidual montana em Lavras, MG. Cerne, Lavras, v. 8, n. 2, p.1-16, 2002.

EMBRAPA_EMPRESA BRASILEIRA DE PESQUISA AGROPECUÁRIA. Manual de análises químicas de solos, plantas e fertilizantes. Embrapa: Brasília, 2009. $627 \mathrm{p}$.

GODINHO, T. O.; CALDEIRA, M. V. W.; COLIMAN, J. P.; PREZOTTI, L. C.; WATZLAWICK, L. F.; AZEVEDO, H. C. A.; ROCHA, J. H. T. Biomassa, macronutrientes, e carbono orgânico na serapilheira depositada em trecho de floresta Estacional Semidecidual Submontana, ES. Scientia Forestalis, Piracicaba, v. 41, n. 7, p. 131-144, 2013.

GOLLEY, F. B. Ciclagem de nutrientes em um ecossistema de floresta tropical úmida. 10. ed. USP: São Paulo, 1978. 256 p.

HOLANDA, A. C.; FELICIANO, A. L. P.; FREIRE, F. J.; SOUZA, F. Q.; FREIRE, S. R. O.; ALVES, A. R. Aporte de Serapilheira e Nutrientes em uma Área de Catinga. Ciência Florestal, Santa Maria, v. 27, n. 2, p. 621-633, 2017. DOI: http://dx.doi.org/10.5902/1980509827747

HOPKINS, B. Vegetation of the Olokemeji Forest Reserve, Nigeria: IV. The litter and soil with special reference to their seasonal changes. Journal of Ecology, v. 54, n. 3, p. 687-703, 1966. https://dx.doi.org/10.2307/2257811

KIM, Y.; KNOX, R. G.; LONGO, M.; MEDVIGY, D.; HUTYRA, L. R.; PYLE, E. H.; WOFSY, S. C.; BRAS, R. L.; MOORCROFT, P. R. Seasonal carbon dynamics and water fluxes in an Amazon rainforest. Global change biology, v. 18, n. 4, p. 01-11, 2012. DOI: https://dx.doi.org/10.1111/j.1365-2486.2011.02629.x

LEITOLD, V.; MORTON, D. C.; LONGO, M.; SANTOS, M. N.; KELLER, M.; SCARANELLO, M. El Niño drought increased canopy turnover in Amazon forests. New Phytologist, v. 219, n. 3, p. 959-971, 2018. DOI: https://dx.doi.org/10.1111/nph.15110

MARTINS, S. V. Ecologia de florestas tropicais do Brasil. 2. ed. Viçosa: UFV, 2012. 371 p.
MARTINS, W. B. R.; FERREIRA, G. C.; SOUZA, F. P.; DIONÍSIO, L. F. S.; OLIVEIRA, F. A. Deposição de serapilheira e nutrientes em áreas de mineração submetidas a métodos de restauração florestal em Paragominas, Pará. Floresta, v. 48, n. 1, p. 37-48, 2018a. DOI: http://dx.doi.org/10.5380/rf.v48i1.49288

MARTINS, W. B. R.; VALE, R. L.; FERREIRA, G. C.; ANDRADE, V. M. S.; DIONÍSIO, L. F.; RODRIGUES, R. P.; OLIVEIRA, G. M. P. Litterfall, litter stock and water holding capacity in post-mining forest restoration ecosystems, Eastern Amazon. Revista Brasileira de Ciências Agrárias, Recife, v. 13, n. 3, p. 1981-1997, 2018b. DOI: http://dx.doi.org/10.5039/agraria.v13i3a5546

MOURA, M. M. S.; COSTA, G. B. R.; PALÁCIO, H. A. Q.; NETO, J. R. A.; BRASIL, J. B. Produção de Serapilheira e suas Frações em área de Caatinga no Semiárido Tropical. Revista Brasileira de Gestão Ambiental e Sustentabilidade, João Pessoa, v. 3, n. 5, p. 199-208, 2016. DOI: http://dx.doi.org/10.21438/rbgas.030509

OLSON, J. S. Energy storage and the balance of producers and decomposers in ecological systems. Ecology, v. 44, n. $2, \quad$ p. 322-331, 1963. DOI: https://dx.doi.org/10.2307/1932179

OURIQUE, L. K.; SILVA, R. O.; SOUZA, C. A. S.; NOGUCHI, H.; SANTOS, J.; HIGUCHI, N. Relação da produção de serapilheira com incremento em diâmetro de uma floresta madura na Amazônia Central. Scientia Florestalis, Piracicaba, v. 44, n. 112, p. 875-886, 2016. DOI: http://dx.doi.org/10.18671/scifor.v44n112.09

PEREIRA, G. H. A.; JORDÃO, H. C. K.; SILVA, V. F. V.; PEREIRA, M. G. Litter and nutrient flows in tropical upland forest flooded by a hydropower plant in the Amazonian basin. Science of the Total Enviroment, v. 572, n. 1, p. 157-168, 2016. DOI: https://dx.doi.org/10.1016/j.scitotenv.2016.07.177

PEREIRA, P. N.; MARTINS, W. B. R. ANDRADE, V. M. S.; OLIVEIRA, F. A. Influência da remoção de serapilheira no teor de fósforo e potássio na Amazônia Oriental. Revista Brasileira de Ciências Agrárias, Recife, v. 12, n. 3, p. 380-385. DOI: http://dx.doi.org/10.5039/agraria.v12i3a5458

SANCHES, L.; VALENTINI, C. M. A.; BIUDES, M. S.; NOGUEIRA, J. S. Dinâmica sazonal da produção e decomposição de serrapilheira em floresta tropical de transição. Revista Brasileira de Engenharia Agrícola e Ambiental, v. 13, n. 2, p. 183-189, 2009. DOI: http://dx.doi.org/10.1590/S1415-43662009000200012

SHANKS, R.; OLSON, J. S. First year breakdown of leaf litter in Southern Appalachia. Forest Science, Bethesda, v. 134, n. 3473, p. 194-195, 1961. DOI: https://dx.doi.org/10.1126/science.134.3473.194

SILVA, H. F.; BARRETO, A. B.; SOUSA, G. T. O.; AZEVEDO, G. B.; GAMA-RODRIGUES, E. F.; OLIVEIRA, F. G. R. B. Decomposição de serapilheira foliar em três sistemas florestais no Sudoeste da Bahia. Revista Brasileira de Biociências, Porto Alegre, v. 12, n. 3, p. 164-172, 2014.

SILVA, W. T. M.; LEONARDO, F. A. P.; SOUTO, J. S.; SOUTO, P. C.; LUCENA, J. D. S.; MEDEIROS NETO, P. H. Deposição de Serapilheira em Áreas de Caatinga no Núcleo de Desertificação do Seridó. Agropecuária Científica no Semiárido, Mossoró, v. 12, n. 4, p. 383390, 2016. 